\title{
Bounds for Lifetime Maximization with Multiple Sinks in Wireless Sensor Networks
}

\author{
Vahid Shah-Mansouri and Vincent W.S. Wong \\ Department of Electrical and Computer Engineering \\ The University of British Columbia, Vancouver, Canada \\ e-mail: $\{$ vahids, vincentw $\} @$ ece.ubc.ca
}

\begin{abstract}
In wireless sensor networks, the reliability can be increased when data is sent to multiple sinks. Data information transmitted to each sink can be considered as a commodity. The objective is to determine the route between each source and sink such that the lifetime of the commodity is maximized. In this paper, we derive the upper and lower bounds for the lifetime maximization problem with multiple sinks. Simulation results show that these bounds are tight for large scale wireless sensor networks.
\end{abstract}

\section{INTRODUCTION}

The recent advances in Micro-Electro-Mechanical Systems (MEMS) technology have accelerated the development of wireless sensor networks (WSNs). WSNs have a wide range of applications, including environmental monitoring, smart building, medical care, and battlefield applications [1]. Since most of the sensor nodes are battery powered, one of the design objectives is to prolong the lifetime of these sensor nodes. Various energy-aware algorithms have been proposed in the literature (e.g., [2], [3]).

The network lifetime in a WSN can be defined as the time at which the first node runs out of its energy. Based on this definition, various algorithms have been proposed to maximize the network lifetime. Chang and Tassiulas formulated the maximum lifetime routing problem as a linear programming problem [4]. In [5], Madan and Lall proposed the partially distributed and fully distributed algorithms to solve the linear maximum lifetime problem in WSNs.

For some of the applications in WSNs (e.g., patients' monitoring, battlefield surveillance), the reliability of the information is also an important design issue [1]. There are several ways to increase the reliability of sending data information from the source to the sink. At the physical layer, the reliability of the links can be increased via the use of forward error correction techniques. At the network layer, one technique is to let each source send its data via multiple paths to the sink [6]. At the transport layer, reliable transport protocols can be used [7].

Another way to increase the reliability is to let each source either choose a sink for transmission or send the data to a number of sinks simultaneously. The latter approach can increase the chance that at least one sink has received the data information. To this end, Lin and Wen [8] proposed a joint scheduling and data aggregation heuristic in order to minimize the total power consumption in a multi-sink WSN.
In [9], Oyman and Ersoy used multiple sinks to reduce the energy consumption of WSN. The network is partitioned into clusters. Each cluster has a sink. The source nodes send data to the corresponding sink in their cluster. In [10], Kalantari and Shayman proposed a vector field formulation to obtain power-efficient routing for multi-sink WSNs. In this scheme, each partition in the WSN has one sink and the source only sends data to the sink within its partition.

The data information transmitted to sink $k$ can be considered as commodity $k$. Our goal is to determine the route between each source and sink such that the lifetime of each commodity is maximized. In this paper, we derive the upper and lower bounds for the lifetime maximization problem with multiple sinks. Simulation results show that these bounds are tight when the number of nodes in the WSN is large.

The rest of the paper is organized as follows: In Section II, we first summarize the maximum lifetime problem formulation with one sink. We then extend the problem formulation to the multi-sink scenario. In Section III, the upper and lower bounds of the lifetime maximization problems are presented. We also describe a distributed implementation algorithm based on dual decomposition. Results are presented in Section IV. Conclusions are given in Section V.

\section{SySTEM MODEL}

\section{A. Maximum Lifetime Routing Problem with One Sink}

We first introduce the notations. Let $V$ denote the set of sensor nodes in a WSN, $N_{i}$ denote the set of neighbors of node $i \in V$. Let $E_{i}$ denote the initial energy of node $i$, and $p_{i j}$ denote the average power consumed for transmission from node $i$ to node $j$. Let $T_{i}(\mathbf{x})$ denote the lifetime of node $i$ under data flow vector $\mathbf{x}=\left\{x_{i j}\right\}$. We have,

$$
T_{i}(\mathbf{x})=\frac{E_{i}}{\sum_{j \in N_{i}} p_{i j} x_{i j}}
$$

Let $S_{i}$ denote rate of data generated by node $i$, and $x_{i j}$ denote the aggregated rate from node $i$ to neighboring node $j$. The data to the sink $S_{\text {sink }}=-\sum_{i \in V, i \neq \operatorname{sink}} S_{i}$. Sensor nodes are assumed to use time division multiple access (TDMA) for data transmission. Links which interfere with each other are not scheduled simultaneously. The maximum transmission rate and the maximum fraction of time allocated to each link are constant. The capacity of link $(i, j)$ is denoted by $R_{i j}$. 
Given $S_{i}, E_{i}, p_{i j}$, and $R_{i j}$, the objective in the maximum lifetime routing problem is to maximize the time that the first node runs out of its energy, subject to the flow conservation constraints. The lifetime routing problem can be formulated as the following linear programming problem [4], [5]:

$$
\begin{array}{ll}
\text { minimize } & q \\
\text { subject to } & \sum_{j \in N_{i}}\left(x_{i j}-x_{j i}\right)=S_{i}, \quad \forall i \in V \\
& \sum_{j \in N_{i}} p_{i j} x_{i j} \leq q E_{i}, \quad \forall i \in V \\
& 0 \leq x_{i j} \leq R_{i j}, \quad \forall i \in V, \forall j \in N_{i}
\end{array}
$$

where $q$ is the upper bound on the inverse of the lifetime of all the nodes in the network. The optimal value (minimal value) is denoted by $q^{\star}$ and is achieved at an optimal solution $\mathrm{x}^{\star}$.

\section{B. Lifetime of a Commodity}

For a WSN with multiple sinks, we assume that each source transmits its information to all of the sinks in order to increase the reliability. Let $C$ denote the set of sinks and $|C|$ denote the total number of sinks. The data that is transmitted to sink $k \in C$ is the commodity $k$. Let $x_{i j}^{k}$ denote the data rate from node $i$ to node $j$ for commodity $k$. The aggregate data rate from node $i$ to $j$ is $\sum_{k \in C} x_{i j}^{k}$. The lifetime of node $i$ under data flow vector $\mathbf{x}=\left\{x_{i j}\right\}$ becomes:

$$
T_{i}(\mathbf{x})=\frac{E_{i}}{\sum_{j \in N_{i}} p_{i j} \sum_{k \in C} x_{i j}^{k}}
$$

The lifetime of commodity $k \in C$ can be defined as:

$$
T^{k}(\mathbf{x})=\min \left\{T_{i}(\mathbf{x}) \mid i \in V \text { and } \sum_{j \in N_{i}} x_{i j}^{k}>0\right\}
$$

In the above definition, we only consider those sensor nodes that carry traffic for commodity $k$. We call node $i$ as the bottleneck node for commodity $k$ when $T_{i}(\mathbf{x})=T^{k}(\mathbf{x})$. For the lifetime routing problem with multiple sinks, the objective is to maximize the lifetime of each commodity. In the next section, we propose two optimization problems which can determine the upper and lower bounds of the maximum lifetime of each commodity.

\section{Bounds of Lifetime MaXimization Problems}

\section{A. Upper Bound Problem}

Consider the following linear programming problem for commodity $k$ in a WSN:

$$
\begin{array}{ll}
\text { minimize } & q_{u}^{k}\left(x_{i j}^{k}-x_{j i}^{k}\right)=S_{i}^{k}, \quad \forall i \in V \\
& \sum_{j \in N_{i}} p_{i j} x_{i j}^{k} \leq E_{i} q_{u}^{k}, \quad \forall i \in V \\
& j_{j \in N_{i}}, \quad \forall i \in V, \forall j \in N_{i}^{k}
\end{array}
$$

where $q_{u}^{k}$ is the inverse of the network lifetime when only commodity $k$ is present in the WSN, $S_{i}^{k}$ denotes the rate of data generated by node $i$ for commodity $k$, and $R_{i j}^{k}=R_{i j} /|C|$ is the capacity for commodity $k$ on link $(i, j)$. We assume that the data rate is much smaller than the link capacity (i.e., $S_{i}^{k} \ll R_{i j}^{k}$ ). The data of commodity $k$ to the $\operatorname{sink} k$ is $S_{\operatorname{sink}}^{k}=$ $-\sum_{i \in V, i \neq \operatorname{sink}} S_{i}^{k}$.

Let $q_{u}^{k \star}$ denote the optimal value of problem (5). When the aggregate traffic from other commodities are also taken into account, the power consumed in each sensor node will either increase or remain the same. Consequently, the lifetime of each commodity will decrease. Thus, $1 / q_{u}^{k \star}$ is the upper bound of the lifetime of commodity $k$. In other words,

$$
T^{k}(\mathbf{x}) \leq \frac{1}{q_{u}^{k \star}}, \quad \forall k \in C
$$

The equality holds when the bottleneck node of commodity $k$ carries data for commodity $k$ only.

\section{B. Lower Bound Problem}

To obtain the lower bound of the lifetime of each commodity, we consider the following scenario: Each intermediate node needs to relay traffic for all commodities in the set $C$. Let $H_{k}$ denote the set of sensor nodes that are within the two-hop neighborhood of sink $k \in C$. Those sensor nodes that belong to $H_{k}$ only relay data to sink $k$. Each sink node $k \in C$ does not relay traffic that is destined for other sink nodes. Consider the following optimization problem for commodity $k$ :

$$
\begin{array}{ll}
\text { minimize } & q_{l}^{k} \\
\text { subject to } & \sum_{j \in N_{i}}\left(x_{i j}^{k}-x_{j i}^{k}\right)=S_{i}^{k}, \quad \forall i \in V \\
& \sum_{j \in N_{i}} \omega_{i}^{k} p_{i j} x_{i j}^{k} \leq E_{i} q_{l}^{k}, \quad \forall i \in V \\
& 0 \leq x_{i j}^{k} \leq R_{i j}^{k}, \quad \forall i \in V, \forall j \in N_{i}
\end{array}
$$

where

$$
\omega_{i}^{k}= \begin{cases}|C|, & \text { if } i \notin H_{k}, \\ 1, & \text { if } i \in H_{k}, \\ M, & \text { if } i \in H_{m}, m \neq k, m \in C\end{cases}
$$

The value of $M$ is chosen as a large number (i.e., $M>>|C|$ ). This ensures that the two-hop neighboring nodes of sink $k$ do not carry traffic for other sinks. For large scale WSNs, we assume that sinks are not within the neighborhood of each other.

Let $q_{l}^{k \star}$ denote the optimal value of problem (6). The value $1 / q_{l}^{k \star}$ is the lower bound of the lifetime of commodity $k$. This can be explained as follows: Each intermediate node $i$ allocates $1 /|C|$ of its initial energy to relay traffic for each commodity $k \in C$. Suppose that node $i$ is the bottleneck node of commodity $k$. If other commodities do not use node $i$ to relay their traffic, then node $i$ can allocate a larger portion of its energy to transmit data for commodity $k$. Thus, the lifetime of commodity $k$ can be increased. In other words,

$$
T^{k}(\mathbf{x}) \geq \frac{1}{q_{l}^{k \star}}, \quad \forall k \in C
$$

The equality holds when all commodities relay traffic via the bottleneck node of commodity $k$. 


\section{Distributed Implementation}

In this section, we describe a distributed algorithm for the lower bound problem (i.e., problem (6)). Due to space limitation, the distributed algorithm for the upper bound problem (i.e., problem (5)) will not be discussed. It can be derived in a similar manner.

The technique that we use is based on dual decomposition [11]. This technique has also been used in [5]. Problem (6) is referred to as the primal problem. In order to decompose the primal problem into several sub-problems, we first replace the term $q_{l}^{k}$ by $\sum_{i \in V}\left(q_{i}^{k}\right)^{2}$. An additional constraint $q_{i}^{k}=q_{j}^{k}$ (for all $i \in V, j \in N_{i}$ ) is included to make sure that all $q_{i}^{k}$ 's are equal. After that, a regularization term $\delta \sum_{i \in V} \sum_{j \in N_{i}}\left(x_{i j}^{k}\right)^{2}$ is added to ensure the uniqueness of the solution, and $\delta$ is a small number. The lower bound problem (6) now becomes:

$$
\begin{aligned}
\operatorname{minimize} & \sum_{i \in V}\left(q_{i}^{k}\right)^{2}+\delta \sum_{i \in V} \sum_{j \in N_{i}}\left(x_{i j}^{k}\right)^{2} \\
\text { subject to } & \sum_{j \in N_{i}}\left(x_{i j}^{k}-x_{j i}^{k}\right)=S_{i}, \quad \forall i \in V \\
& \sum_{j \in N_{i}} \omega_{i}^{k} p_{i j} x_{i j}^{k} \leq E_{i} q_{i}^{k}, \quad \forall i \in V \\
& q_{i}^{k}=q_{j}^{k}, \quad \forall i \in V, \forall j \in N_{i}, \\
& 0 \leq x_{i j}^{k} \leq R_{i j}^{k}, \quad \forall i \in V, \forall j \in N_{i}
\end{aligned}
$$

Problem (7) is a convex optimization problem. To solve problem (7), we use the dual decomposition approach. We first introduce the Lagrange multipliers $\left(\nu^{k}\right.$ and $\left.\gamma^{k}\right)$ for the equality constraints in (7). The other constraints are local constraints in each node and do not need to be relaxed. The Lagrangian $L\left(\mathbf{q}^{k}, \mathbf{x}^{k}, \nu^{k}, \gamma^{k}\right)$ is:

$$
\begin{aligned}
L\left(\mathbf{q}^{k}, \mathbf{x}^{k}, \nu^{k}, \gamma^{k}\right)= & \sum_{i \in V}\left(\left(q_{i}^{k}\right)^{2}+\delta \sum_{j \in N_{i}}\left(x_{i j}^{k}\right)^{2}\right) \\
& +\sum_{i \in V} \nu_{i}^{k}\left(\sum_{j \in N_{i}}\left(x_{i j}^{k}-x_{j i}^{k}\right)-S_{i}\right) \\
& +\sum_{i \in V} \sum_{j \in N_{i}} \gamma_{i j}^{k}\left(q_{i}^{k}-q_{j}^{k}\right) \\
= & -\sum_{i \in V} \nu_{i}^{k} S_{i}+\sum_{i \in V}\left(\left(q_{i}^{k}\right)^{2}\right. \\
& +\delta \sum_{j \in N_{i}}\left(x_{i j}^{k}\right)^{2}+q_{i}^{k} \sum_{j \in N_{i}}\left(\gamma_{i j}^{k}-\gamma_{j i}^{k}\right) \\
& \left.+\sum_{j \in N_{i}} x_{i j}^{k}\left(\nu_{i}^{k}-\nu_{j}^{k}\right)\right) .
\end{aligned}
$$

From the Lagrangian, the dual function and the dual problem can be defined. A subgradient algorithm can be used to solve the dual problem. We use the following distributed algorithm to solve problem (7).
Distributed algorithm for each source $i \in V$ and each commodity $k$ :

Given $\nu^{k}(t), \gamma^{k}(t)$, and the local information $\left(p_{i j}, E_{i}, R_{i j}\right)$, each node $i$ updates the variables $x_{i j}^{k}(t)$ and $q_{i}^{k}(t)$ by solving the following problem:

$$
\begin{array}{cl}
\operatorname{minimize} & \left(q_{i}^{k}(t)\right)^{2}+\delta \sum_{j \in N_{i}}\left(x_{i j}^{k}(t)\right)^{2} \\
& +q_{i}^{k}(t) \sum_{j \in N_{i}}\left(\gamma_{i j}^{k}(t)-\gamma_{j i}^{k}(t)\right) \\
& +\sum_{j \in N_{i}} x_{i j}^{k}(t)\left(\nu_{i}^{k}(t)-\nu_{j}^{k}(t)\right) \\
\text { subject to } & \sum_{j \in N_{i}} \omega_{i}^{k} p_{i j} x_{i j}^{k}(t) \leq E_{i} q_{i}^{k}(t) \\
& 0 \leq x_{i j}^{k}(t) \leq R_{i j}^{k}, \quad \forall j \in N_{i}
\end{array}
$$

The Lagrange multipliers $\nu_{i}^{k}(t)$ and $\gamma_{i j}^{k}(t)$ at each iteration $t$ are updated according to the following equations:

$$
\nu_{i}^{k}(t+1)=\nu_{i}^{k}(t)-\alpha(t)\left(S_{i}-\sum_{j \in N_{i}}\left(x_{i j}^{k}(t)-x_{j i}^{k}(t)\right)\right),
$$

and

$$
\gamma_{i j}^{k}(t+1)=\gamma_{i j}^{k}(t)-\alpha(t)\left(q_{j}^{k}(t)-q_{i}^{k}(t)\right), \forall j \in N_{i}
$$

where $\alpha(t)$ is a positive diminishing step size and is chosen as $\frac{1}{t+1}, x_{i j}^{k}(t)$ and $q_{i}^{k}(t)$ are solutions of problem (8).

In summary, the values of $x_{i j}^{k}$ and $q_{i}^{k}$ are updated in each iteration $t$ at each node based on (8). The values of Lagrange multipliers $\nu_{i}^{k}(t)$ and $\gamma_{i j}^{k}(t)$ are updated based on (9) and (10). Each node then exchanges the updated values of the Lagrange multipliers with its neighbors.

\section{Performance Analysis}

In this section, we present the results of the upper and lower bounds of the lifetime maximization problems. In the experiment, we gradually increase the number of nodes in the network, starting with 50 and with incremental steps of 10. Sensor nodes are randomly deployed in a square area. In order to ensure that the density of the nodes is the same, the size of the coverage area is increased proportionally when we increase the number of nodes in the network. The maximum transmission radius of each node is assumed to be 1 unit. The number of sources in all of the topologies is equal to 40. All sources are randomly chosen from the nodes in the network, and each topology has three sinks.

We assume a deterministic path loss model. The power consumed for transmission of one bit from node $i$ to node $j$ (i.e., $p_{i j}$ ) is $\beta_{1}+\beta_{2} d^{4}$, where $d$ is the physical distance. We choose $\beta_{1}=1$ and $\beta_{2}=0.1$. For the regularization terms, we select $\delta=10^{-5}$. Each source generates $100 \mathrm{bytes} / \mathrm{sec}$ of information. The initial energy of each source node $E_{S}$ is 30 $\mathrm{J}$ while the initial energy of each intermediate node $i$ (i.e., $E_{i}$ ) is $10 \mathrm{~J}$. 


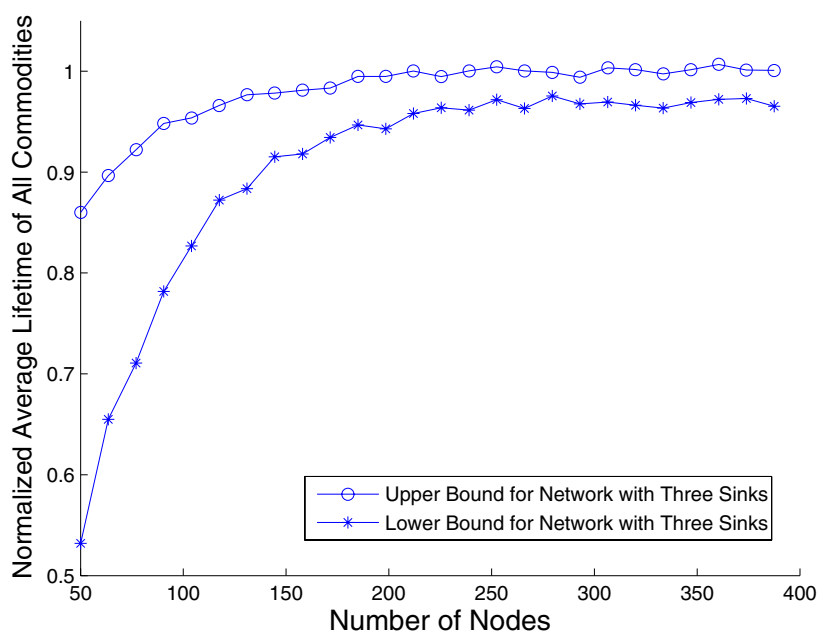

Fig. 1. Normalized average lifetime of all commodities for the network with three sinks.

Figure 1 shows the results of the upper bound and lower bound of the lifetime for the network. Results are averaged over 1000 simulation runs. The lifetime is the average lifetime of all commodities in the network normalized with respect to the last value of the upper bound of the network with three sinks. When the number of nodes is increased, the bottleneck node of each commodity is more likely to be close to the sink and only carries the information that belongs to that particular sink. In this case, the optimal values obtained for commodity $k$ in problems (5) and (6) become close. Thus, these bounds are tight for large scale wireless sensor networks.

In the next simulation experiment, we determine the number of iterations required for the distributed algorithm to converge for the lower bound problem. The number of nodes in the WSN is 400. Results from Figure 2 show that it takes 500 iterations for the algorithm to converge within $10 \%$ of the optimal value. It takes approximately 20,000 iterations for the algorithm to converge within $1 \%$ of the optimal value.

\section{CONCLUSIONS}

The reliability of the information can be increased when the data is sent to multiple sinks in a WSN. In this paper, we formulated two optimization problems and derived the upper and lower bounds for the lifetime maximization problem with multiple sinks. We also described a distributed algorithm for implementation in large scale WSNs. Simulation results show that these bounds are tight when the number of nodes in the WSN is large. For future work, we plan to determine the optimal value for the lifetime maximization problem with multiple sinks.

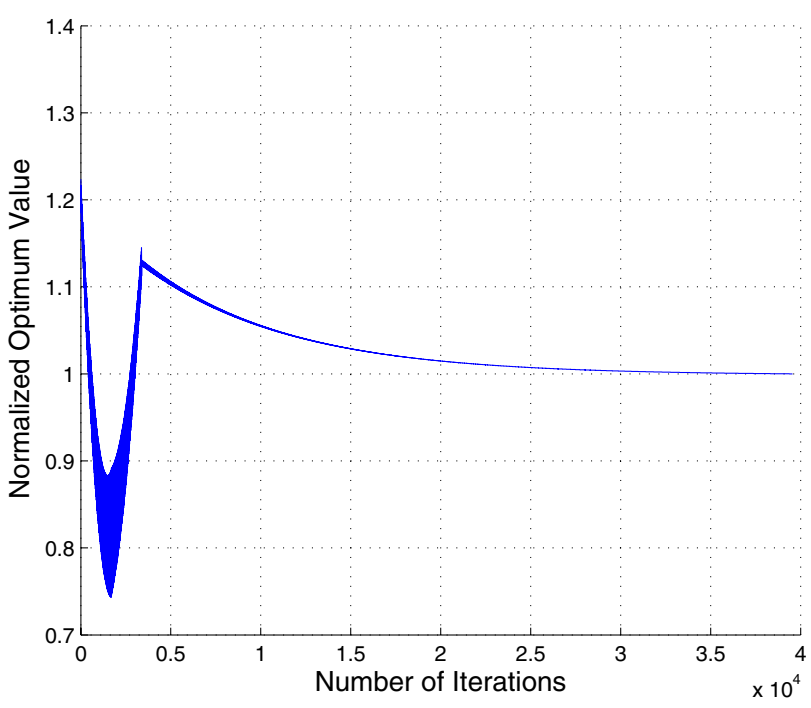

Fig. 2. Normalized optimum value of the problem for one of commodities for the lower bound problem for a WSN with 400 nodes.

\section{ACKNOWLEDGEMENT}

This work is supported by the Natural Sciences and Engineering Research Council of Canada (NSERC).

\section{REFERENCES}

[1] I. F. Akyildiz, W. Su, Y. Sankarasubramaniam, and E. Cayirci, "Wireless sensor networks: A survey," Computer Networks (Elsevier) Journal, vol. 38, pp. 393-422, 2002.

[2] V. Rodoplu and T. Meng, "Minimum energy mobile wireless networks," IEEE J. on Selected Areas in Communications, vol. 17, pp. 1333-1344, Aug. 1999.

[3] A. Goldsmith and S. Wicker, "Design challenges for energy-constrained ad hoc wireless networks," IEEE Trans. on Wireless Communications, vol. 9, pp. 8-27, Aug. 2002.

[4] J. Chang and L. Tassiulas, "Routing for maximum system lifetime in wireless ad-hoc networks," in Proc. of Allerton Conference Communication, Control, and Computing, Urbana, Illinois, Sept. 1999.

[5] R. Madan and S. Lall, "Distributed algorithms for maximum lifetime routing in wireless sensor networks," IEEE Trans. on Wireless Communications, vol. 5, pp. 2185-2193, Aug. 2006.

[6] F. Ye, G. Zhong, S. Lu, and L. Zhang, "Gradient broadcast: A robust data delivery protocol for large scale sensor networks," Wireless Networks Journal (WINET), vol. 11, pp. 285-298, 2005.

[7] O. B. Akan and I. F. Akyildiz, "Event-to-sink reliable transport in wireless sensor networks," IEEE/ACM Trans. on Networking, vol. 13, pp. 1003 - 1016, Oct. 2005.

[8] F. Lin and Y. Wen, "Multi-sink data aggregation routing and scheduling with dynamic radii in WSNs," IEEE Communications Letters, vol. 10, pp. 692- 694, 2006.

[9] E. I. Oyman and C. Ersoy, "Multiple sink network design problem in large scale wireless sensor networks," in Proc. of IEEE ICC, Paris, France, June 2004.

[10] M. Kalantari and M. Shayman, "Design optimization of multi-sink sensor networks by analogy to electrostatic theory," in Proc. of IEEE WCNC, Las Vegas, Nevada, April 2006.

[11] S. Boyd and L. Vandenberghe, Convex Optimization. Cambridge University Press, 2004. 